\title{
NON-ARCHIMEDEAN METRICS IN TOPOLOGY
}

\section{J. DE GROOT}

1. We ask under which conditions a topological space is metrizable in a non-archimedean way, i.e. under which conditions one can describe the topological structure of the space by defining a suitable metric which satisfies-instead of the triangle axiom-the stronger axiom

$$
\rho(\xi, \eta) \leqq \max [\rho(\xi, \zeta), \rho(\eta, \zeta)] .
$$

We shall find the following necessary and sufficient conditions:

I. the space is metrizable (cf. Nagata [1], Smirnof [2]),

II. the space is strongly 0-dimensional.

Property II means that any two closed disjoint sets in the space can be separated (by the empty set). We shall prove furthermore that the conditions I and II are equivalent to the following topological properties: the space is a Hausdorff space having a 0 -dimensional NS-base.

We call an open base of the space an NS-base, if it is the sum of a countable number of locally finite families (a family of open sets is locally finite, if any point of the space is contained in an open set which intersects at most a finite number of sets of the family). If the sets of this base are both open and closed, we call it a 0-dimensional NS-base.

In a metric space $M$ a non-archimedean metric can therefore be introduced (in a topologically equivalent way) if and only if $M$ is strongly 0 -dimensional. This settles a problem raised by A. F. Monna [4] some years ago. The question remains unsolved as to whether the condition of strong 0 -dimensionality may be replaced by a weaker form of 0-dimensionality (any point and closed set, mutually disjoint, can be separated). Of course the answer is positive in the case of separable metric spaces, for both notions are then equivalent (cf. $[3$, p. 15]). However, it seems probable to me that these notions are not equivalent in general metric spaces (cf. [3, appendix], for the case of more general topological spaces). Does there exist a (weakly) 0 -dimensional metric space in which two certain disjoint closed sets cannot be separated? In such a space it would be impossible to introduce a non-archimedean metric.

Received by the editors June 2, 1955. 
The non-archimedean metrics are of some interest in topology, for, if they can be introduced, the handling of a number of problems becomes much simpler (essentially because the $\epsilon$-neighborhoods of two different points are either disjoint or identical).

In $\$ 2$ we give- because of its simplicity-a solution of the characterization theorem for the well-known case of separable metric spaces. To prove a characterization theorem in general metric spaces, we define in $\S 3$, for any cardinal number $\beta$, a generalized Hilbert space $N^{\beta}$ such that an arbitrary strongly 0 -dimensional metrizable space can be embedded in $N^{\beta}$ if $\beta$ is suitably chosen. This Hilbert space, once defined, yields to a proof of Theorem I, suggested by the methods used by Urysohn and Smirnof. In $\$ 5$ we give the characterization theorem its final form.

2. The separable case. Let $S$ be a topological space satisfying

$\left(1^{\circ}\right) S$ is a Hausdorff space (or more generally, a $T_{0}$-space).

$\left(2^{\circ}\right)$ In $S$ exists a countable base consisting of both open and closed sets $\left\{B_{i}\right\},(i=1,2, \cdots)$.

To each point $x \in S$ let there be attached an ordered sequence of integers $\left(x_{1}, x_{2}, \cdots, x_{i}, \cdots\right)$ such that $x_{i}=0$ if $x \notin B_{i}$ and $x_{i}=1$ if $x \in B_{i}$. The distance $\rho(x, y)$ of two points is defined by

$$
\rho(x, y)=\max _{n}\left\{\frac{1}{n}\left|x_{n}-y_{n}\right|\right\} .
$$

It is easy to see that this distance function is non-archimedean. Since, conversely, any non-archimedean separable metric space obviously satisfies the properties $\left(1^{\circ}\right)$ and $\left(2^{\circ}\right)$, the separable nonarchimedean metrizable spaces are therefore topologically equivalent with the Hausdorff spaces having a countable base consisting of both open and closed sets.

This gives, incidentally, a simple solution of the metrization problem in the case of 0-dimensional separable spaces.

3. A generalized non-archimedean Hilbert space $N^{\beta}$. Let $\beta \geqq \boldsymbol{N}_{\theta}$ be the cardinal of a set of indices $\{\alpha\}$. The set of ordered pairs $\{n \alpha\}$, where $n$ runs through the natural numbers, has the same cardinal $\beta$. A point of $N^{\beta}$ is any characteristic function $\xi_{n \alpha}=\xi(n \alpha)$ (which therefore assumes only the values 0 and 1 ) defined on the set $\{n \alpha\}$. The distance of two functions $\xi(n \alpha)$ and $\eta(n \alpha)$ is defined by

$$
\rho(\xi, \eta)=\max _{n, \alpha}\left\{\frac{1}{n}|\xi(n \alpha)-\eta(n \alpha)|\right\} .
$$


Obviously $N^{\beta}$ is semi-metric.

To prove (1), for different points $\xi$ and $\eta$ and a certain $n=m$, we note that

$$
\rho(\xi, \eta)=\frac{1}{m}|\xi(m \alpha)-\eta(m \alpha)|=\frac{1}{m},
$$

and therefore

$$
\xi(m \alpha)=1 \text { or } 0, \quad \eta(m \alpha)=0 \text { or } 1
$$

Now, obviously,

$$
\max \{|\xi(m \alpha)-\zeta(m \alpha)|,|\eta(m \alpha)-\zeta(m \alpha)|\}=1,
$$

from which (1) follows.

4. ThEOREM I. A topological space is non-archimedeanly metrizable if, and only if, it is a Hausdorff space (or even a $T_{0}$-space) having a 0 dimensional NS-base.

Proof. A non-archimedean metric space $M$ is of course a Hausdorff space. To define a 0 -dimensional NS-base in $M$, let $\left\{U_{\epsilon}\right\}$ ( $\epsilon$ arbitrary but fixed) be a covering of $M$ in disjoint (open and closed) $\epsilon$-neighborhoods of certain points. The existence of such a covering follows from an easy transfinite induction, considering the fact that the $\epsilon$-neighborhoods of two different points are either disjoint or identical. Therefore, the sum of an arbitrary number (finite or transfinite) of disjoint $\epsilon$-neighborhoods is open and closed and has a distance $\geqq \epsilon$ from its complement in $M$. Now the sum of the countable number of families $\left\{U_{1 / n}\right\}(n=1,2, \cdots)$ is obviously the required 0 -dimensional NSbase.

Conversely, let $H$ be a $T_{0}$-space, in which a countable number of locally finite families $\left\{U_{n \alpha}\right\}(n=1,2, \cdots)$ is given, such that the system of all both open and closed $U_{n \alpha}$ is a base of $H$. Let $\beta$ be the cardinal of the set $\{\gamma\}$ of all possible indices $\gamma=n \alpha$. We put $U_{n(n \alpha)}$ $=U_{n \alpha}, U_{n(m \alpha)}=0(n \neq m)$. The families $\left\{U_{n \gamma}\right\}$ remain locally finite. We define for each $x \in H$ and each pair $n \gamma$ a function $\xi(x)$

$$
\xi_{n \gamma}(x)=\left\{\begin{array}{l}
1, \text { if } x \in U_{n \gamma}, \\
0, \text { if } x \in H \backslash U_{n \gamma} .
\end{array}\right.
$$

We map any $x \in H$ on $f(x) \in N^{\beta}$ with $f(x)=\left\{\xi_{n \gamma}(x)\right\}$.

The mapping $f$ is one-to-one, since, to each pair of different points $x$ and $y$, there corresponds a $U_{n \alpha}$ containing $x$ and not containing $y$. Therefore $\xi_{n \alpha}(x)=1, \xi_{n \alpha}(y)=0$, and $f(x) \neq f(y)$. 
The $\operatorname{map} f$ is continuous. Let $O_{\epsilon}$ be an $\epsilon$-neighborhood with $\epsilon=1 / m$ ( $m$ sufficiently large) in $N^{\beta}$ of a point $f(x)=\left\{\xi_{n \alpha}(x)\right\}$. For $n \leqq m$ there is only a finite number of sets $U_{n \alpha}$ which intersect a certain neighborhood $O(x)$ of $x$. Among these $U_{n \alpha}$, there are two types: the $U_{k \alpha}$ which contain $x$, and the $U_{l \alpha}$ not containing $x$. Let

$$
V(x)=\left[\bigcap_{k \leqq m} U_{k \alpha} \backslash \bigcup_{l \leqq m} U_{l \alpha}\right] \cap O(x) .
$$

$V(x)$ obviously is an open neighborhood of $x$. We shall show

$$
f(V(x)) \subset O_{\text {. }}
$$

Indeed for any point $y \in V(x)$ and $n \leqq m: \xi_{n \alpha}(x)-\xi_{n \alpha}(y)=0$, since $x$ and $y$ are both in $U_{n \alpha}$ or in its complement. Now the relation

$$
\rho(f(x), f(y))=\rho\left(\xi_{n \alpha}(x), \xi_{n \alpha}(y)\right) \leqq \frac{1}{m+1}
$$

is trivial, since (2) contains the factor $1 / n$.

The map $f$ is open. Consider an open set $O \subset H$ and let $x \in O$. There is a certain $U_{s \alpha}$ with $x \in U_{s \alpha} \subset O$. Therefore $\xi_{s \alpha}(x)=1$. Now, if the condition $\rho(f(x), f(y))<1 / s$ is fulfilled for a certain point $y \in H$, it follows from (2) that $\xi_{s \alpha}(y)=1$, which gives us $y \in U_{s \alpha} \subset O$. The set $f(O)$ is therefore open in $f(H)$.

The mapping $f$ being topological, thus introduces the required nonarchimedean metric in $H$ by considering $f(H)$ instead of $H$.

5. TheOREM II. A metrizable space is non-archimedeanly metrizable if, and only if, it is strongly 0-dimensional.

COROLlaRY. $A$ non-archimedean metric can be introduced in a metric space if, and only if, the metric space is strongly 0-dimensional.

Proof. According to $[1 ; 2]$ a topological space $R$ is metrizable if, and only if, $R$ is regular and has an NS-base $\left\{O_{n \alpha}\right\} .{ }^{1}$ Let $O=O_{n \alpha}$ be an arbitrary open set of this NS-base. If $R$ is strongly 0 -dimensional, $O$ may be considered as the sum of a countable number of mutually disjoint both open and closed sets

$$
O=\bigcup_{i=1}^{\infty} U_{i}
$$

1 In the definition of an NS-base in $\$ 1$ we are considering "families," whereas Smirnof [2] speaks of "coverings," i.e. families which are coverings. The difference is irrelevant, since any family becomes a (locally finite) covering by adding the entire space as an element to each family. 
To prove this, introduce a metric in $R$ and consider the $\epsilon$-neighborhoods $V_{\epsilon}$ with $\epsilon=1 / n(n=1,2, \cdots)$ of $R \backslash O$

$$
V_{\epsilon}=V_{\epsilon}(R \backslash O) \text {. }
$$

If the disjoint open and closed sets $U_{i} \subset O(i \leqq n-1)$ are already defined, we proceed to define $U_{n} \subset O$ in the following way. The closed disjoint sets $R \backslash O$ and

$$
S=\left[R \backslash V_{1 / n}\right] \cup\left[\bigcup_{i=1}^{n-1} U_{i}\right]
$$

can be separated. Let $T$ be a both open and closed set containing $S$ and contained in $O$. Then

$$
U_{n}=T \backslash \bigcup_{i=1}^{n-1} U_{i}
$$

In this way any $O_{n \alpha}$ is decomposed in a countable number of disjoint both open and closed sets $U_{\text {in } \alpha}(i=1,2, \cdots)$. The countable number of families $\left\{U_{\text {in } \alpha}\right\}=\left\{U_{k \alpha}\right\}(k=1,2, \cdots)$, originated by enumerating the pairs $k=(i, n)$ is obviously a 0 -dimensional NS-base. Now we can apply Theorem I. $R$ is therefore non-archimedeanly metrizable.

Conversely, it is easy to see that any non-archimedean metric space is strongly 0-dimensional by introducing a 0-dimensional NS-base (which is possible according to Theorem I) and using the normality of a metric space.

Added in proof. The present manuscript was prepared several years ago. Since, a short abstract, containing some applications as well, has been published by $\mathrm{H}$. de Vries and the author (Ind. Math. vol. 17 (1955) pp. 222-224). In the meantime two papers on dimension theory in metric spaces have become known to the author ( $M$. Katětov, Čz. Mat. Ž. Tsjechoslov. Mat. Zj. vol. 2 (77) (1952) pp. 333-368 (in Russian) and K. Morita, Math. Ann. vol. 128 (1954) pp. 350-362). Now it is clear-and this has already been pointed out by A. H. Stone (in litt.) -that the metrization theorems of Nagata and Smirnof can be applied to certain results on zero-dimensional spaces contained in these papers (see e.g. $\$ 10$ in Morita's paper; all spaces in this paper are assumed to be metric), which yields an alternate (but not essentially simpler) proof of our results (strongly zerodimensional is called zero-dimensional in these papers; however, the problem mentioned in 1 remains unsolved).

Recently, J. Nagata (Proc. Jap. Ac. vol. 32 (1956) pp. 237-243), 
leaning heavily on dimension-theoretical results in general metric spaces, proved an interesting far reaching generalization of our results by characterizing the (finite) dimension of a metrizable space by means of the possibility of assigning a certain metric to the space (this metric becomes non-archimedean in the case of dimension zero).

\section{REFERENCES}

1. J. Nagata, On a necessary and sufficient condition of metrizability, J. Inst. Polyt. Osaka vol. 1 (1950) pp. 93-100.

2. J. Smirnof, $A$ necessary and sufficient condition for metrizability of a topological space, Doklady Akademii Nauk SSSR. (N.S.) vol. 77 (1951) pp. 197-200.

3. W. Hurewicz and H. Wallman, Dimension theory, Princeton, 1941.

4. A. F. Monna, Remarques sur les métriques non-archimédiennes, I, Neder. Akad. Wetensch. vol. 53 (1950) pp. 470-481.

University of AMSTERDAM 